\title{
Traffic Data: Bluetooth Sensors vs. Crowdsourcing-A Comparative Study to Calculate Travel Time Reliability in Calgary, Alberta, Canada
}

\author{
Shahram Tahmasseby \\ Transportation Planning, The City of Calgary, Calgary 2500, Canada
}

\begin{abstract}
The City of Calgary did a comparative study between two techniques of traffic data collection: Bluetooth sensors and crowdsourcing, for measuring travel time reliability on two goods movement corridors in Calgary, Alberta. To estimate travel time and speed, we used the output of BluFAX sensors, which were operated by monitoring Bluetooth signals at several points along a roadway. On the other hand, TomTom historical traffic data were extracted from the TomTom Traffic Stats portal. To calculate travel time reliability, we applied the buffer index, and the planning time index recommended by FHWA (Federal Highway Administration). The Bluetooth traffic data were presumed as the benchmark in this study. Unlike the TomTom traffic data, the data provided by the Bluetooth technology met the minimum recommended sample size requirement, although data processing was time consuming and impractical for long study periods. Our study results showed that crowdsourcing technique can be a viable alternative and provide travel time reliability estimates with a reasonable accuracy, when there are adequate numbers of records registered. However, the TomTom sample sizes in Calgary were not large enough to provide a statistically reliable method of providing travel time indices. Further researches may verify the accuracy of crowdsourcing technologies for travel time studies.
\end{abstract}

Key words: Bluetooth, BluFAX, travel time reliability, TomTom, buffer time index, GPS (global positioning system) based traffic data, crowdsourced traffic data.

\section{Background and Study Motivation}

Calgary in the province of Alberta, Canada is an intersection of two major transportation corridors (Fig. 1):

- the Trans-Canada Highway which is a transcontinental federal-provincial highway system that travels through all 10 provinces of Canada between its Pacific Ocean and Atlantic Ocean coasts to the west and east, respectively. It is, along with the Trans-Siberian Highway and Australia's Highway 1, one of the world's longest national highways with the main route spanning 8,030 km (4,990 miles) (Fig. 1);

- the Queen Elizabeth II Highway (known as Deerfoot Trail in Calgary), which features 21 interchanges, stretches $50 \mathrm{~km}$ and is Alberta's busiest

Corresponding author: Shahram Tahmasseby, Ph.D., P.Eng., research fields: transportation systems engineering and intelligent transportation systems. E-mail: Shahram.Tahmasseby@calgary.ca. highway with traffic volumes ranging between 27,000 and 158,000 vehicles per day depending on the location [1].

Calgary is a key distribution centre of Asia-Pacific related imports and exports. Trucking accounts for $46 \%$ of imports and $64 \%$ of exports. Over the last 14 years, the number of registered commercial vehicles has been growing faster than the population. This indicates the growing importance of commercial travel within Calgary. According to Alberta Infrastructure and Transportation's Office of Traffic Safety [2], between 2005 and 2007, the number of registered commercial vehicles in Calgary increased from 88,386 to 110,500 , (25\%), while Calgary's population increased by $7 \%$. Commercial vehicles made over 265,000 trips a day in 2006. Out of those vehicle trips, nearly $80 \%$ had origins and destinations within the city limits. 


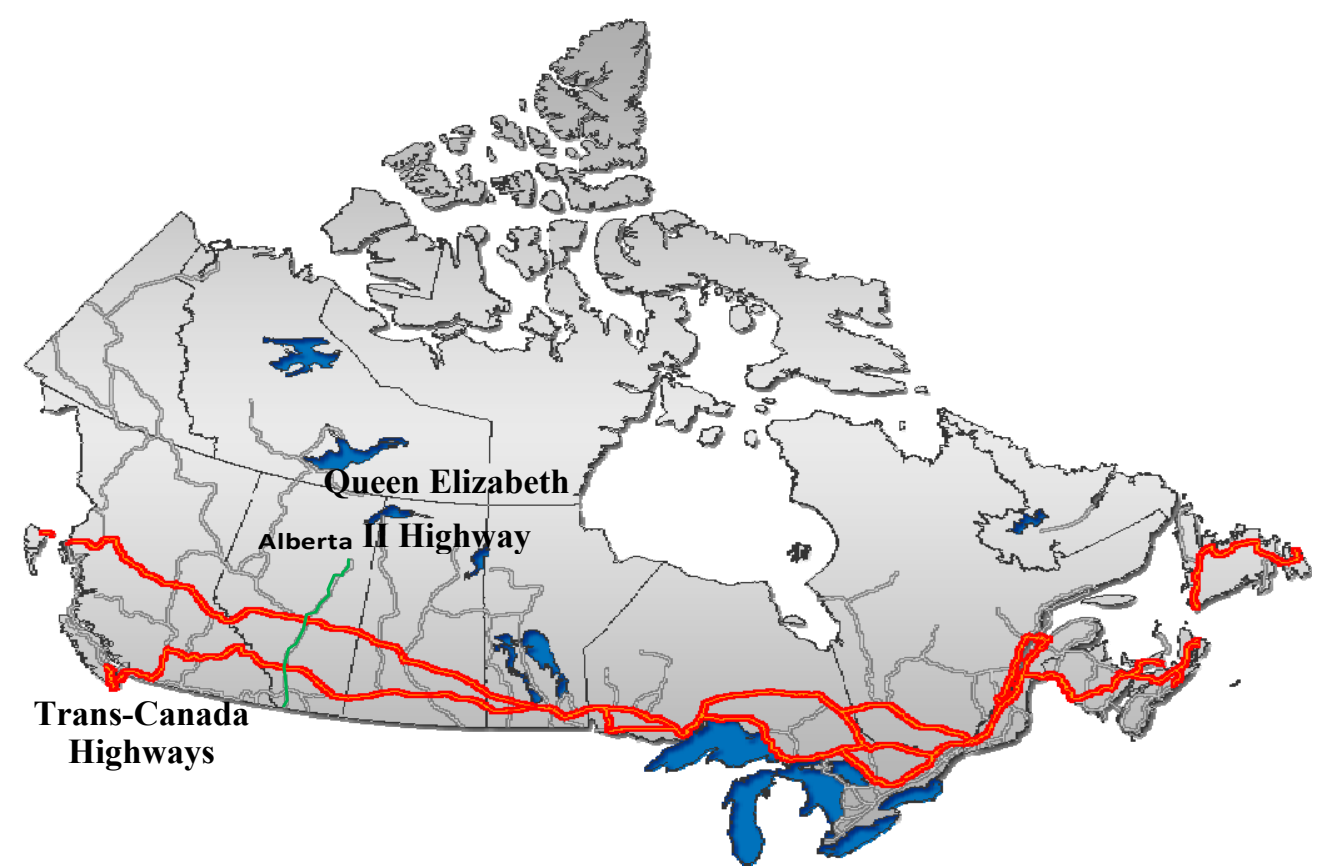

Fig. 1 The nationwide Trans-Canada Highway (stretched over Canada from east coast to west coast) and the provincial Queen Elizabeth II Highway in Alberta (north-south).

The goods movement corridors are identified based on the primary goods movement network map prepared by the city (Fig. 2). The important corridors are identified according to the percentage of traffic consisting of trucks on the primary goods movement corridors and thus ranking the corridors.

The MDP (Municipal Development Plan) [3] and the CTP (Calgary Transportation Plan) [4] both recognized the important role of goods movement in Alberta economy in 2009. A safe, well-organized and connected goods movement network contributes to a prosperous economy.

The Monitoring and Reporting Program provides a mechanism through which the goals, objectives and policies of the MDP and CTP are being evaluated. The programme identifies several citywide indicators that are relevant to the implementation of these plans. The travel time reliability on the goods movement network is one of the citywide benchmarks monitored by the programme. By estimating the travel time reliability on selected goods movement corridors over time, the effects of network improvements and the impacts of congestion and delay on commercial vehicle movement can be measured and alleviated as required.

Currently, most road authorities use loops, pneumatic tubes (hoses), magnetic sensors, cameras or infrared sensors to measure traffic performance. Although, these traditional systems are proven methods of collecting traffic data, they each also have a number of drawbacks and shortcomings such as:

- The initial cost can be excessive because these systems may need the installation of hardware alongside the road or directly on the road surface;

- Maintenance costs can be significant as well due to extreme weather conditions or vandalism;

- Most of these traditional systems are not able to precisely quantify speed and travel times over segments and can only provide spot speeds at point locations with a reasonable degree of accuracy;

- While camera systems with automatic license plate recognition can measure travel times, they are incapable of measuring variations in speed between two camera locations or provide local-level speed measurements along the path;

- The reliability of camera and loop systems depends on calibration and validation; 
Traffic Data: Bluetooth Sensors vs. Crowdsourcing-A Comparative Study to Calculate Travel Time Reliability in Calgary, Canada

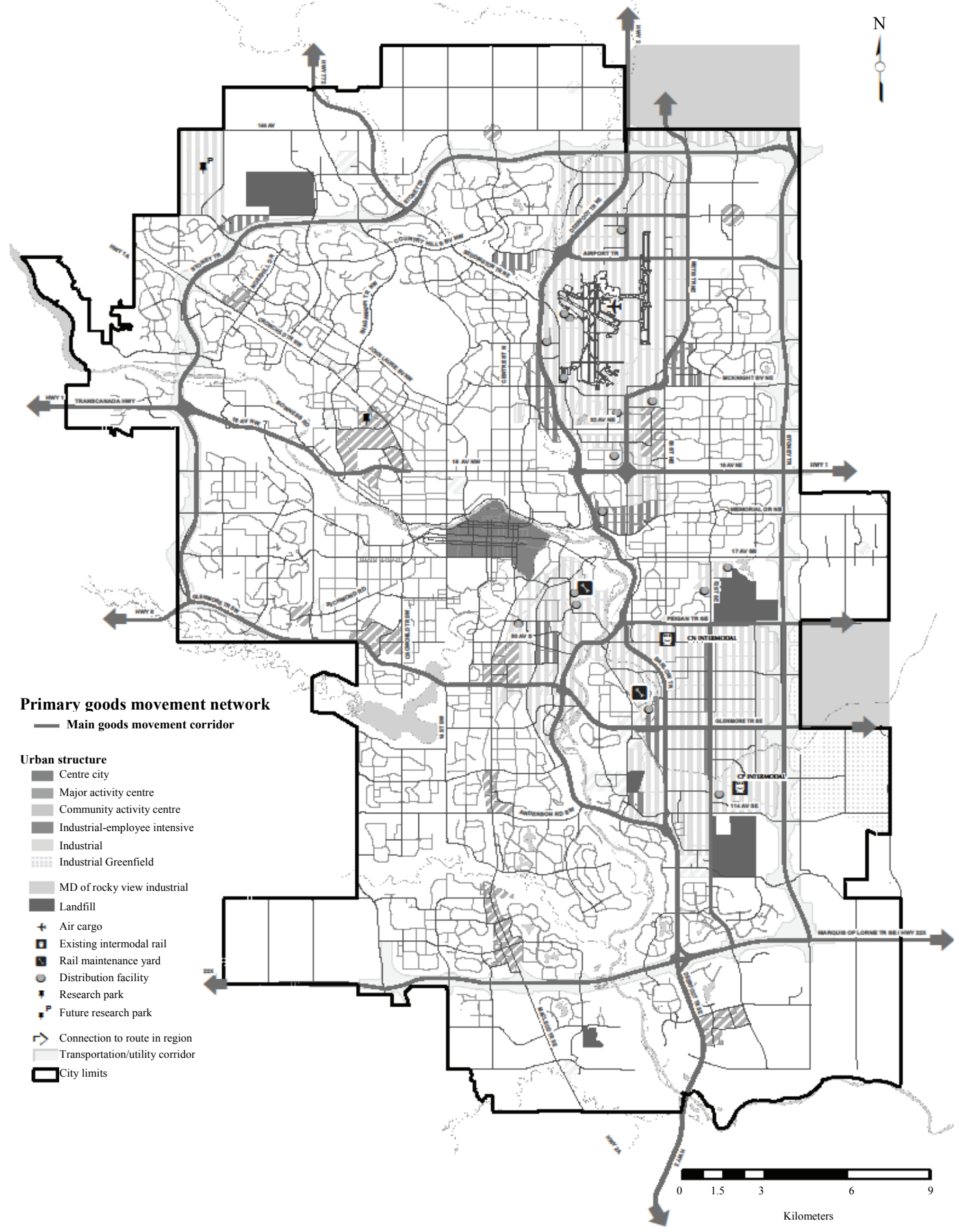

This map represents a conceptual land use structure and transportation networks for the city as a whole.

Fig. 2 The City of Calgary primary goods movement network.

Source: the city of Calgary, ROADS [5]. 
- Automated counts do not provide information and perception on travel behaviors.

Given the aforementioned disadvantages and to fulfill the mandate of the MDP and CTP programmes, the Transportation Department of the City of Calgary [6] has investigated opportunities to use new technologies, including Bluetooth travel time monitoring equipment, as well as real time and historical traffic data provided by crowdsourcing techniques. The city envisages developing the methodologies for travel time and speed measurements with the following characteristics:

- repeatable from year to year using available data;

- simple and easy to understand;

- cost-effective;

- easily applicable and reliable;

- always feasible given the regions' prolonged and harsh winters.

\section{Bluetooth Technology}

Given the traditional methodologies' shortcomings for travel time and speed measurement and the city's trend to leverage the latest techniques of traffic data collection, the Bluetooth technology could be a preferred alternative to provide a larger sample size of travel time and speed at a lower cost than that is possible with the conventional methods.

Bluetooth is a wireless technology standard for exchanging data over short distances (using short-wave length UHF (ultra high frequency) radio waves in the ISM (industrial, scientific and medical) band from $2.4 \mathrm{GHz}$ to $2.485 \mathrm{GHz}$ ) from fixed and mobile devices and building PANs (personal area networks).

In terms of accuracy, the Bluetooth technology gives an outstanding level of preciseness compared to the other methods, i.e., inductive loops, radar detectors and image processors commonly used to infer travel time, because travel time is directly sampled rather than inferred from the point speed detections.

\section{Crowdsourcing Methodology for Traffic Data Collection}

The wide deployment area of Bluetooth sensors incurs significant costs including personnel time and costs to traffic organizations.

Ironically, crowdsourcing has introduced a new method for measuring speed, travel time and thus road performance measures since the past decade. GPS (global position system)-based data record, GSM (global system for mobile communication)-based FCD (floating car data), in dash navigation, and other forms of crowdsourced traffic data are practical and handy in this context. Probe devices in moving vehicles, which may be cellular phones, or more commonly GPS devices, provide crowdsourced traffic data. In the context of FCD, as vehicles with probe devices are free to move everywhere on the road network, they are called "floating" probes and are not limited to roadside infrastructure to communicate with the FCD system. Consequently, FCD can measure traffic speeds everywhere probe vehicles are moving. The major advantages of crowdsourcing in comparison to the traditional methods could be outlined as follows:

- capability of measuring speeds and travel times on lengthy stretches as well as the variance in speed along the way with low cost and without degrading traffic safety;

- capability to understand travelers' behavior without conducting travel surveys;

- no need for installation or maintenance of roadside units and devices, saving substantial costs and avoiding any disruptions to traffic flow;

- supporting sustainability and environment;

- providing information which is doable for the whole traffic network and thus not limited to the parts of the network facilitated by data collection instruments;

- changing the trend of fieldwork with office work for the purpose of traffic data collection, resulting in cost savings and thus saving field personnel's time. 
Due to the fact that the quality and accuracy of GPS-based and crowdsourcing technologies are continually improving, it will not be unrealistic to presume that applying these technologies will be dominant in transport authorities in the near future to calculate real-time speed, travel time index, travel time reliability metrics, queue measurements and so on. In other words, they have potentials to be considered as reliable alternatives for traditional methods of travel time studies and may result in significant cost and time savings for traffic and transportation organizations particularly when dealing with tight budgets and limited number of equipment and staff. Nonetheless, the benefits of crowdsourcing may vary and depend on the adequacy as well as accuracy of the data.

\section{Recent Practices in Bluetooth and Crowdsourcing Techniques}

Bluetooth technology as a non intrusive method for traffic data collection has been used for traffic measurement since 2005. When vehicles are carrying detectable Bluetooth devices (i.e., navigators, mobile phones, headsets, etc.), they can be identified by Bluetooth sensors installed at multiple locations along a highway. Bluetooth sensors generally are less costly than other sensor technologies. However, expansion of this technology for traffic monitoring and especially travel time estimation depends on asserting its accuracy and reliability. Outliers are a common problem in travel time studies and the reliability of Bluetooth studies depends on elimination of them [7].

Recently, TTI ${ }^{1}$ conducted a comparative cost analysis for data collection using different techniques, i.e., toll tags, dual loops, magnetometer, private sector, radar and Bluetooth [8]. The total costs (in thousand dollars per mile) for a 5-year monitoring period revealed that the Bluetooth technology is the least costly method compared to the others.

In the I-95 Corridor Coalition's VPP (Vehicle Probe

${ }^{1}$ TTI is the Texas A\&M Transportation Institute in College Station, Texas is the largest transportation research agency in the United States.
Project), BluFAX sensors have been used by the University of Maryland since July 2008 to monitor INRIX $^{2}$ [9] freeway data quality for the I-95 Corridor Coalition VPP $[10,11]$. On this corridor, the Bluetooth technology has set the standard for quality control for outsourced traffic data [10].

In 2010, $\mathrm{MTO}^{3}$ was the first in Canada to deploy Bluetooth units for traffic delay compliance monitoring [12]. Permissible traffic delays are included in the Performance Specifications of MTO's new Design-Build Contracts, which portray the maximum length of time a contractor may delay traffic throughout a construction zone.

In 2011, the City of Calgary conducted travel time and speed studies on three goods movement corridors in the city (Glenmore Trail, Barlow Trail and Deerfoot Trail) by using its BluFAX sensors [13]. Travel times and according speed were measured on the aforementioned roads three times a year (September/October, January/February and May/June), each time 20 weekdays, to provide a comprehensive travel pattern and to include the impacts of seasonal variations on travel time. To estimate the penetration rate, pneumatic tubes were used to count the traffic volume on the abovementioned corridors. The penetration rate and the study results accuracy demonstrated the applicability of BluFAX sensors for short time assignments.

In the context of the applicability of crowdsourcing technology in transportation modeling, Prato et al. [14] present the estimation of the value of congestion and the value of travel time reliability from the observation of drivers' route choice behavior in the greater Copenhagen area in 2011. Car drivers were driving a vehicle equipped with GPS and recorded GPS points were matched to the network of the Danish national

\footnotetext{
${ }^{2}$ INRIX is a SaaS (software as a service) and DaaS (data as a service) company which provides a variety of internet services and mobile applications pertaining to road traffic and driver services.

${ }^{3}$ MTO (Ministry of Transportation of Ontario) is the provincial ministry of the government of Ontario which is responsible for transport infrastructure and related law in Ontario, Canada.
} 
model (LTM (landstrafikmodel)) for constructing a dataset of observed route choices.

TomTom ${ }^{4}$ has recently published a traffic index for more than 160 cities worldwide for the public information for the year of 2013 [15]. The methodology being developed compares travel times during non-congested periods (free flow) with travel times in peak hours. The difference is expressed as a percentage increase in travel time.

INRIX uses a proprietary data fusion engine which has been developed using a Bayesian modeling and proprietary error correction technology to process over 400 crowdsourced data aggregated by the INRIX Smart Driver Network and to generate traffic information accordingly [16, 17]. INRIX collects trillions of bytes of information about roadway speeds from nearly 100 million anonymous mobile phones, a few vehicle manufacturers, trucks, delivery vans and other fleet vehicles equipped with GPS locator devices, Garmin Navigation, Kenwood Traffic Services, etc.. The data collected are processed in real-time, creating performance metrics on analytic tools (https://inrix.ritis.org/analytics/screencast/) by exhibiting, for example, historical traffic speed information, buffer time index, etc., on major freeways, highways and arterials across the US continent. The format of the data appears to be somehow similar to the TomTom historical traffic data and performance metrics.

The MTO in 2012 purchased travel time data from two vendors namely INRIX and TomTom for the province of Ontario for 725 directional freeways, 407 directional arterials and eight ramps within the greater Toronto study area [12, 18]. Also, travel time obtained from Bluetooth receivers was obtained from the ministry for selected arterial road sections and ramps. A comparison was made between and among these three data sources. The study results showed that both TomTom and INRIX data sources were able to provide

\footnotetext{
${ }^{4}$ TomTom NV is a Dutch manufacturer of automotive navigation systems, including both stand-alone units and software for personal digital assistants and mobile telephones.
}

traffic data for both collector and express facilities. However, both sources failed to provide data for high occupancy lanes separate from the general purpose lane. Similarly, the Bluetooth technology is incapable to provide results with a reasonable accuracy given its inherent shortcoming in terms of traffic distinction between close parallel routes.

KMJ Consulting, Inc. conducted a comparative study to evaluate the efficiency and viability of two different methods of travel time data collection on a signalized arterial corridor (Verree Road in Philadelphia, PA (Pennsylvania)), i.e., traditional method of floating test car runs using GPS equipments and the deployment of Bluetooth technology using the BlueTOAD ${ }^{5}$ product $[19,20]$. They observed a number of advantages when using the Bluetooth technology such as offering more realistic approach to measure travel time as experienced by the drivers, the ease of installation and operation, and less inclination for human error. However, they pointed out some disadvantages as well, such as requirement for a certain technology awareness/knowledge to operate the device (installation, downloading data), the large amount of data which can pose analytical challenges, cost, vulnerability to vandalism and the requirement for access to publicly or privately own infrastructure such as utility poles, traffic signal poles, etc. for installation. Nonetheless, the study findings demonstrated that the Bluetooth technology is the most cost effective alternative given the volume of data required.

\section{Bluetooth Technology Widely Used by the City of Calgary}

Given the advantages of the Bluetooth technology in travel time measurement, the City of Calgary's Roads Traffic Division [21] uses data collected from travelers' Bluetooth devices to routinely measure travel time and speed on the city's skeletal and goods movement corridors and thus calculates and publishes travel time

\footnotetext{
${ }^{5} \mathrm{TOAD}$ stands for travel-time origination and destination.
} 
reliability metrics based on travel time observations.

The technology currently being used by the city is "BluFAX sensor" which is a battery-operated, self-contained unit designed by Traffax Inc. to conduct travel time and O-D (origin-destination) studies on freeways, arterials and urban roadways. It is suitable for measuring travel time on both freeways and arterial roads with a variety of modes. The system can collect data from pedestrians, or from any vehicles occupied by individuals carrying Bluetooth-enabled devices as long as the Bluetooth devices are paired.

Furthermore, it is supported by proprietary software (BluSTATS) to process and analyze the BluFAX sensors data [10]. BluSTATS generates segment summary statistics including segment length, number of data points, travel time/speed descriptive statistics (e.g., mean, median, minimum, maximum, percentiles and standard deviations) in a .cvs format suitable for manipulation in 5, 15 and $60 \mathrm{~min}$ time frames. Moreover, it combines segments to constitute alternative paths through the study corridors.

Currently, the city of Calgary has permanent monitoring stations equipped with BluFAX sensors along the Queen Elizabeth II Highway (known as Deerfoot Trail in Calgary), Crowchild Trail and Glenmore Trail (Fig. 3). For travel time studies on other corridors, the city typically installs portable BluFAX sensors as temporary Bluetooth traffic data stations.

As indicated before, the main goal of operating those units is to provide real-time travel time information to the public. The roads operations centre stores the real-time data which are then downloaded and analyzed by the transportation data division to determine the travel time and travel time reliability indices. Fig. 4 exhibits travel time reliability, represented by planning time index, during p.m. peak hours (working days only) in the month of December in 2013 on few segments of abovementioned corridors provided by the city owned stationary sensors. Fig. 4 clearly shows that the planning time index exceeded
$600 \%$ on some segments demonstrating severe traffic congestion in peak hours on the city's skeletal roads and goods movement corridors.

Similar to BlueTOAD's shortcomings observed in the case of Verree Road in Philadelphia, PA, the main challenge of the BluFAX sensors in Calgary is BluSTATS' output formation which creates a huge number of recorded vehicles on high traffic volume corridors. Creation of such enormous records imposes difficulties to traffic data analysts in terms of data processing, manipulation and thus conducting travel time and speed studies. Given the software's (BluSTATS) limited capacity for processing massive data, the data need to be processed in two or three weeks chunks. This process is time consuming and notably demands a great deal of a data analyst's working time. For the sake of practicability and given the limited capacity of the BluSTATS software, it is not possible to calculate travel time reliability metrics on a high traffic volume corridor (e.g., Deerfoot Trail) for the entire year.

The other observed deficiency was frequent interruptions and malfunctions during the operation of the units as the units were aging. This was the case particularly for the portable sensors which resulted in significant gaps and omissions in the traffic database.

Given the challenges of using the BluFAX system, for longer study periods on heavily used roadways, the crowdsourced traffic data for travel time data collection were investigated. A privilege was given to the City of Calgary by TomTom to use its Traffic Stats portal for accuracy check of historical traffic data in the Calgary region. The portal is user-friendly and its users can define routes consisting of a number of segments, study periods (year, month and day) as well as time of the day. Currently, TomTom is providing historical travel time and speed indicators from the year 2008 onwards.

\section{TomTom Historical Traffic Data}

According to a TomTom white paper about historical 
BluFAX units placement

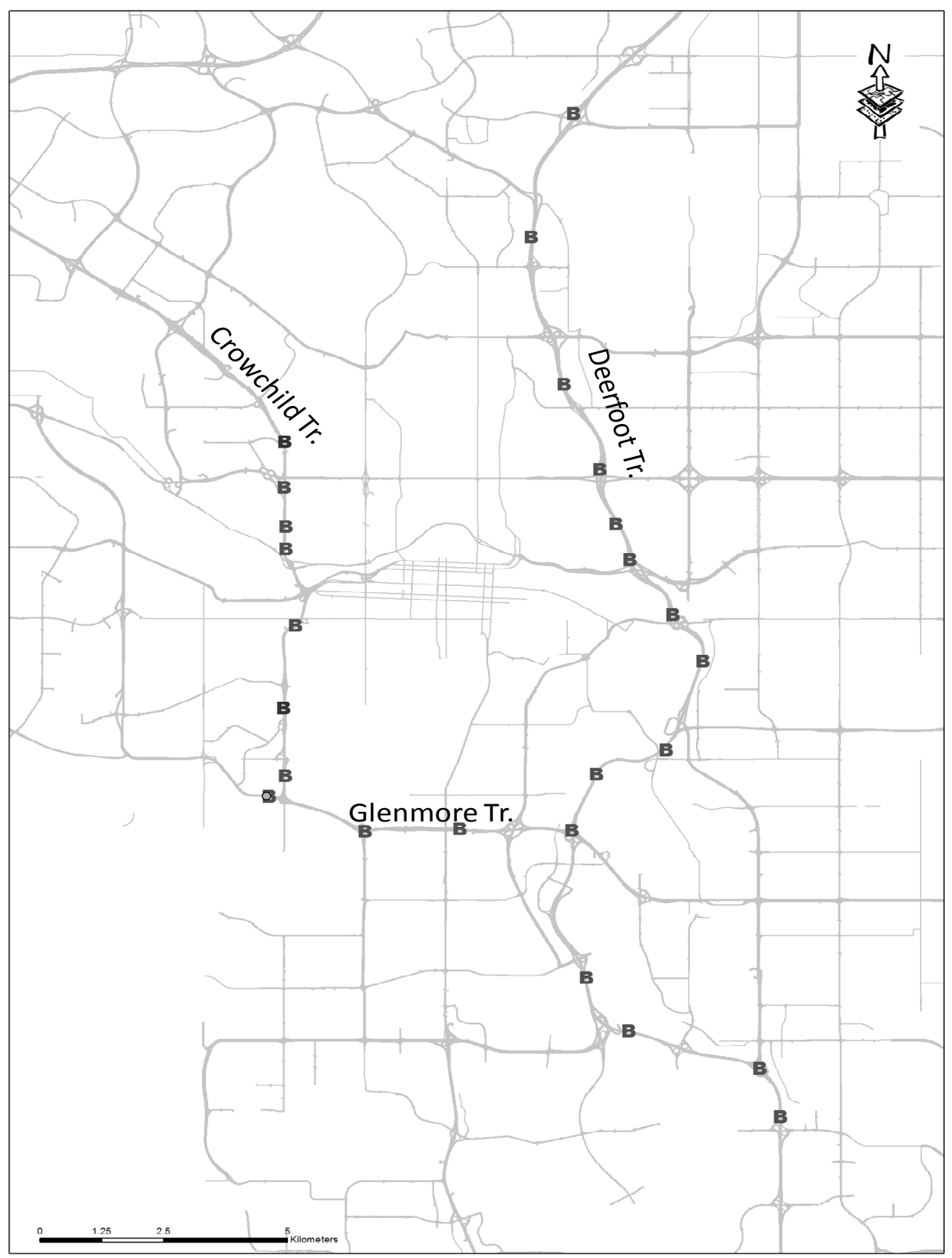

Fig. 3 Permanent BluFAX stations in Calgary, Alberta.

traffic information [22], the traffic data are collected by millions of TomTom navigation device users worldwide who voluntarily agree to share their anonymous usage statistics.

TomTom processes the raw crowdsourced traffic information received from customers in a number of ways in order to protect privacy, filter outliers and create geographic databases which can be queried.
When connecting users' GPS device to their computer, these users report data anonymously for each of their journeys. According to the TomTom aforementioned white paper, the company now has a database containing more than 6 trillion measurements worldwide, collected since 2008 from millions of TomTom users. Every day, numerous new measurements are added to this database and this figure increases exponentially. 


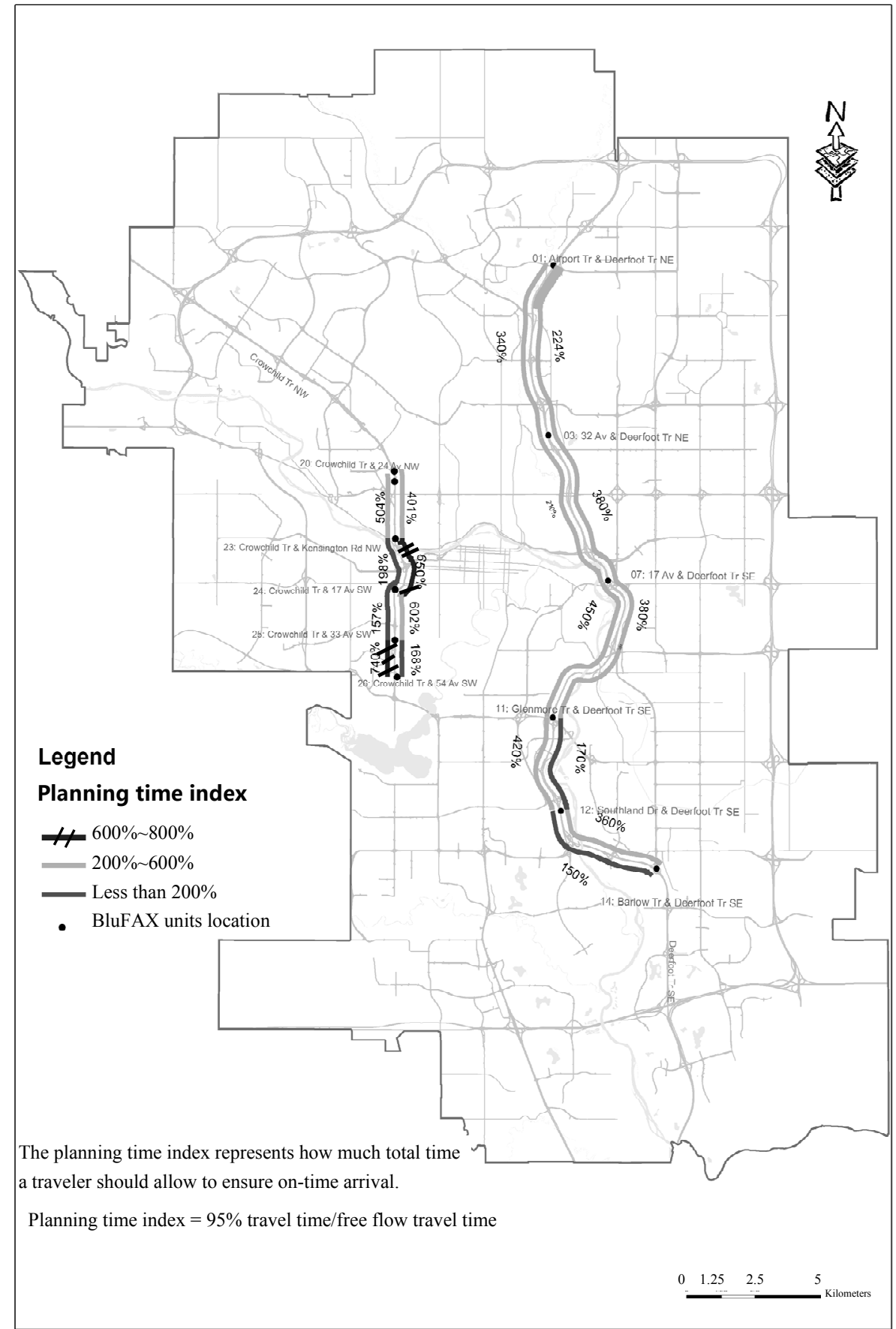

Fig. 4 Travel time reliability index on two skeletal freeways in Calgary.

\subsection{Traffic Stats}

Historical traffic products from TomTom are being delivered on the cloud through a self-service web portal solution, called Traffic Stats. The portal ensures that with a set of inputs (that is routes, dates and times), the user can specify a customized query for their own area or road of interest, their specific days of interest and the time periods in that date range that are of interest (Fig. 5). The results are computed and a report is generated by the portal for downloading or online view. 


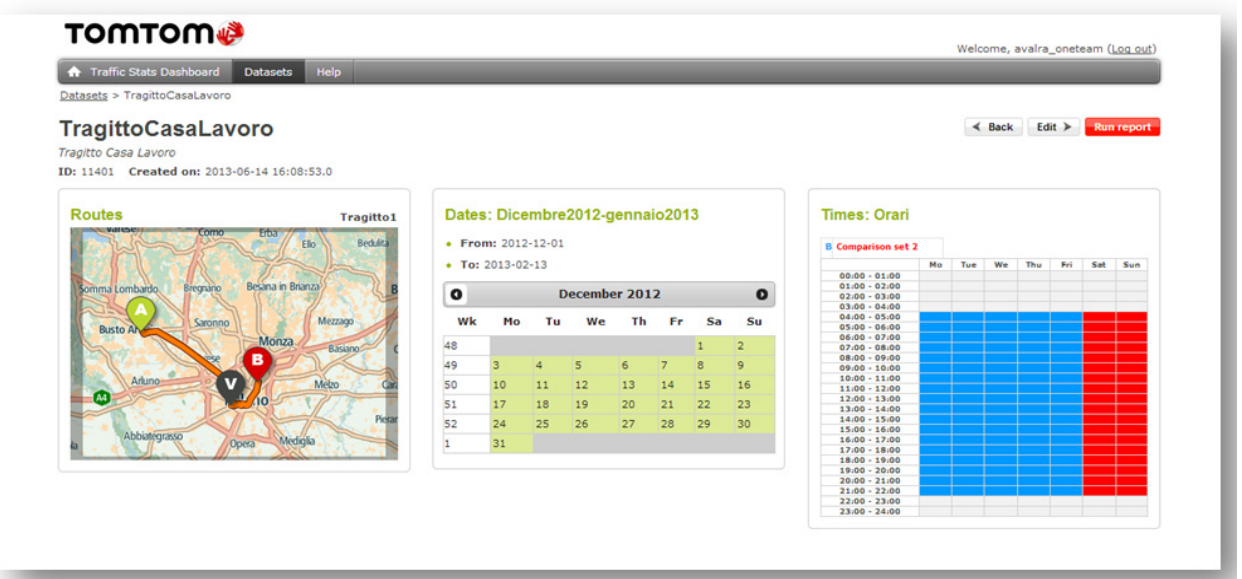

Fig. 5 TomTom Traffic Stats.

Source: TomTom real time traffic data infosheet [22].

The results, which are route-based, are given in three different types of output:

- Excel file;

- KML file format ${ }^{6}$;

- charts.

The Excel file contains the following information:

- length of route;

- sample size of unique vehicles over the entire route (or part of the route);

- arithmetic average travel time across the route;

- arithmetic median travel time across the route;

- harmonic average speed over route;

- travel time ratio (for "comparison set" time period compared to the "base set" time period);

- average travel time across the route, i.e., the 5th percentile, 10th percentile, ..., 90th percentile and 95th percentile.

\section{Comparative Study in the City of Calgary}

In order to evaluate the applicability of TomTom historical traffic data in travel time reliability and speed studies, two case studies were conducted on two congested goods movement corridors (a freeway and an arterial road). The main goal was to examine the efficacy, accuracy and applicability of the

\footnotetext{
${ }^{6} \mathrm{KML}$ is a type of file format used to display geographic data in an Earth browser such as Google Earth, Google Maps and Google Maps for mobile.
}

crowdsourcing technology in travel time reliability and speed studies and whether it could truly supersede the Bluetooth technology in some conditions.

Based on the ITERIS research on sample size, $3 \% \sim 11 \%$ of actual traffic flow in North America carries Bluetooth-enabled devices [23]. A study by the University of Maryland demonstrates that well-placed sensors should provide a $4 \%$ detection rate for roadways of 36,000 ADT (average daily traffic) or more [11]. Young [24] showed even a $2 \%$ match rate on a roadway of 100,000 ADT would provide more than enough hits to accurately generate mean travel times in 5 min intervals. An early test of Bluetooth travel time measurement produced match rates of $1.2 \%$ and $0.7 \%$ [25]. An evaluation of the BlueTOAD system captured approximately $4 \%$ of the traffic stream [19]. Kittelson \& Associates, Inc., obtained match rates of $3 \%$ and $4 \%$ during the a.m. peak and p.m. peak respectively during a 2009 Bluetooth study [26].

Similarly, the penetration rate was estimated from a series of study on Crowchild Trail in the City of Calgary in 2011 when the BluFAX units were initially obtained by the city. The studies showed that the penetration rate was approximately $3 \%$ following the exclusion of outliers and anomalies. BluSTATS, the BluFAX proprietary software, matches Bluetooth MAC (media access control) address between designated pairs of sensor stations, applying robust 
statistical base filtering and segment processing to flag high values of travel time as outliers and to discard MAC IDs not detected at both locations.

Given the previous case studies' findings, it was concluded that the sample size should be at least $3 \%$ of the total vehicle volume within the monitored time period and that a minimum of 20 days of data collection is needed to determine travel time reliability.

Given the constraints in data processing for long-lasting study periods on high volume roadways and the main study focus on working days, we considered 15 working days in an uninterrupted 20-day period (from Oct 1, 2012 to Oct 21, 2012). The total studied segment length of this corridor is approximately $2 \mathrm{~km}$ and the free flow speed is presumed to be the maximum allowed speed of $100 \mathrm{~km} / \mathrm{h}$.

As Fig. 3 illustrates, 15 permanent BluFAX stations are currently operating along this corridor measuring travel times and speeds. These sensors continually detect and record Bluetooth signals as they come in range. Each signal's unique MAC address is recorded alongside date and time of day. By tracking when individual signals reach various points along the route, registration times are determined. By comparing the records from different sensors, the travel time for MAC addresses detected at multiple sensors was computed (Fig. 6). Then, travel time data were clustered into different time periods, such as a.m. peak, p.m. peak and weekday based on the starting time. The average and 95\% travel times were calculated for each segment of the corridor. As indicated before, BluSTATS matches Bluetooth MAC address between designated pairs of sensor stations, discards MAC IDs not detected at both locations and flags high values of travel time as outliers.

\subsection{Case Study-A Segment on the Queen Elizabeth II Highway (Known As Deerfoot Trail in Calgary)}

This corridor is Alberta's busiest highway with traffic volumes ranging between 40,000 and 189,000 vehicles per day [4].

It is an important primary goods movement corridor in the province and connects the City of Calgary to Edmonton (the province capital) as well as forming a critical link in Alberta's north-south trade corridor.

In this case study, we opted for the segment between $\mathrm{S} 2$ and S1 as depicted in Fig. 7. The figure exhibits the study segment which stretches from the Beddington Trail interchange (S2) to Airport Trail exit (S1) in the northbound direction of Deerfoot Trail.

There are two permanent count stations on Deerfoot Trail within the study area and we leveraged those stations for the BluFAX accuracy check. In order to assert the adequacy of the BluFAX sample size and therefore the study accuracy, we compared the number of observations recorded by BluFAX sensors during the aforementioned period to that registered by the permanent count stations along the corridor.

The average daily traffic count in the northbound direction of this corridor is about 56,000 vehicles per day (working days). BluFAX sensors provide adequate number of records (36,191 out of $1,100,000$ vehicles) which exceeds the minimum recommended threshold of $3 \%$ (Table 1). Nonetheless, TomTom's observations for this corridor in this 20 day period were negligible (only 532 records) and did not meet the minimum sample size requirement. One reason could be the TomTom navigation system is not widely used in Alberta by drivers. Moreover, truck drivers may be familiar with their routes or use alternative navigating

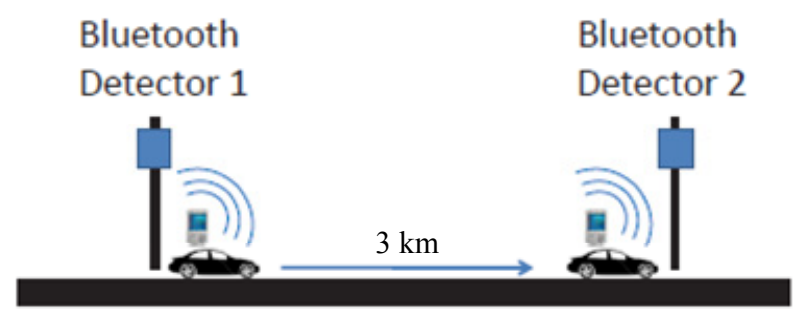

Time: 9:13:26 a.m.

Time: 9:15:58 a.m.

Travel time: $2: 32 \mathrm{~min}$
Speed: $71 \mathrm{~km} / \mathrm{h}$

Fig. 6 Leveraging the Bluetooth technology to measure travel time and speed on a corridor. 


\section{Deerfoot Trail 2 BluFAX units}

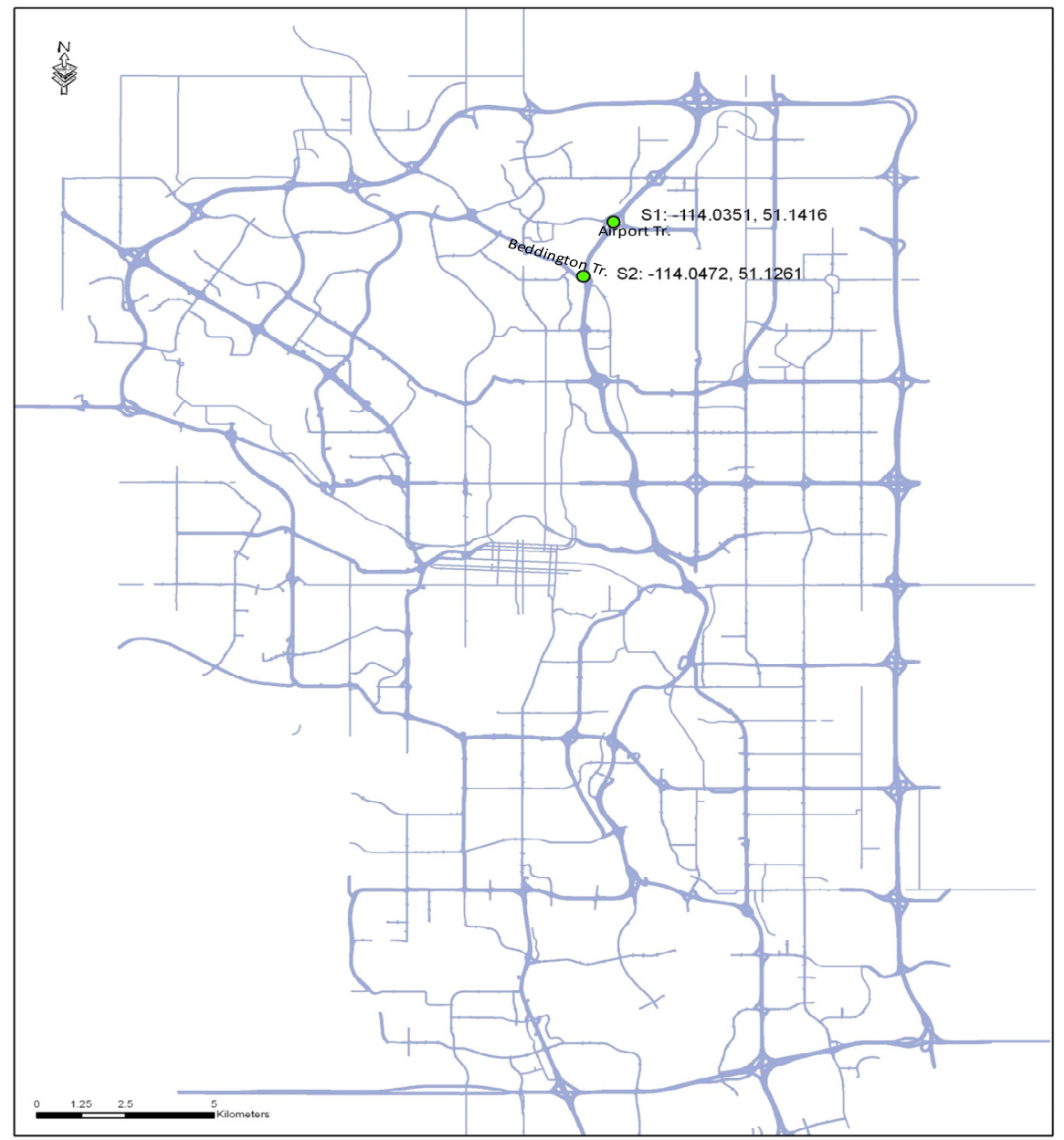

Fig. 7 The study segment on Deerfoot trail between Beddington Trail and Airport Trail exit (northbound direction).

systems such as Garmin, Google Maps or MapQuest which are widely used in Canada. Given the inadequate number of TomTom records, it would be expected that the travel time reliability study yields a biased result.

\subsection{Travel Time Reliability Indicators}

The average speed and travel time reliability indicators are periodically published as "customer mobility indicators" as part of the CTP and MDP monitoring and reporting programs $[3,4]$ as well as included as performance measures for the transportation department. The travel time reliability measures, which have been adopted and included in the MDP/CTP monitoring and reporting programs, are expressed as follows:

- the $95 \%$ travel time;
- the buffer index;

- the travel time index;

- the planning time index.

For more information on these indices, we can refer to the Office of Operations of FHWA ${ }^{7}$ and strategic highway research program [27, 28].

Table 1 outlines the cumulative travel time reliability indices as well as the average speed for the studied corridor in the 20-day study period over $24 \mathrm{~h}$ during weekdays and excluding the weekends.

The travel time reliability indices indicate that the drivers need to consider $38.9 \%$ extra travel time to ensure an on time arrival based on the BluFAX study

${ }^{7}$ FHWA (the Federal Highway Administration) is a division of the United States Department of Transportation that specializes in highway transportation. 
outcome. However, TomTom only indicates that 5.4\% extra travel time is required. Regarding the travel time indices, the figures show that the ratio of the actual travel time to the theoretical travel time on Deerfoot Trail stays close to $95 \%$ with the Bluetooth methodology and TomTom estimation shows 91.7\%. Finally, both TomTom and BluFAX outcomes show that the average speed during weekdays has exceeded the maximum allowed speed on this corridor. It is noteworthy to mention that this section of Deerfoot Trail, which lies in the very northern part of the city, usually does not suffer from heavy traffic congestion and the level of service is expected to be on average either A or B on weekdays.

In the context of comparison of TomTom to BluFAX and thus evaluating the accuracy of TomTom results, the travel time reliability figures resulted from applying BluFAX and TomTom techniques are not identical, although the difference in terms of the travel time index is more or less negligible (Table 1). However, the outcomes show that the two applied techniques yielded quite dissimilar figures when it comes to the planning time index.

As the outcomes demonstrate, the inadequacy of observations provided by TomTom degrades the travel time reliability and the average speed figures.

\subsection{Case Study 2-52nd Street}

Similarly, we conducted a comparative travel time study using two abovementioned data sources on a main arterial goods movement corridor, 52nd St., (Fig. 8) in Calgary form the last week of October 2012 to the first week of November 2012.

The total length of the studied corridor was approximately $7.5 \mathrm{~km}$ and the observed free flow speed was about $65 \mathrm{~km} / \mathrm{h}(5 \mathrm{~km} / \mathrm{h}$ higher than the maximum allowed speed). In order to have a clear picture of the adequate BluFAX sample size, we applied the pneumatic tube technology twice (on two different days) to have a rough estimation of regular traffic flow in the working days for the study period. The average daily traffic count for the southbound of this corridor is about 15,000 vehicles per day.

The procedure of Bluetooth travel time monitoring on this corridor began by mounting a few portable sensors along the route for the study period (Fig. 8). These sensors continually detected and recorded Bluetooth signals as they came in range, similar to the permanent BluFAX stations in the previous case study.

To ensure having adequate sample size along the corridor, the corridor was split into four segments. BluFAX sensors were placed at locations between the segments (Fig. 8) to capture data from vehicles that did not travel the entire path on this corridor. The data from these segments were then aggregated into a single dataset for the entire corridor.

Table 2 shows that unlike the case study of Deerfoot Trail, the number of TomTom records on the 52nd St. improved, although it was still inadequate and did not meet the minimum sample size criterion.

Table 1 Comparative travel time reliability study on Deerfoot Trail (24 h).

\begin{tabular}{llll}
\hline Indicator & BluFAX & TomTom & Difference \\
\hline Number of observations & 36191 & 532 & - \\
Detection rate & $3.3 \%$ & Negligible & - \\
Weekday average travel time (min:s) & $1: 06$ & $1: 07$ & $1.5 \%$ \\
Weekday 95\% travel time (min:s) & $1: 36$ & $1: 20$ & $16 \%$ \\
Weekday buffer time (95th percentile-average travel time) (s) & 30 & 13 & $56.6 \%$ \\
Weekday buffer index (\%) & $45.5 \%$ & $14.9 \%$ & $67.2 \%$ \\
Weekday travel time index (\%) & $95.5 \%$ & $91.7 \%$ & $4.0 \%$ \\
Weekday planning time index (\%) & $138.9 \%$ & $105.4 \%$ & $24.1 \%$ \\
Weekday average speed $(\mathrm{km} / \mathrm{h})$ & 101.3 & 107.5 & $-6.1 \%$ \\
\hline
\end{tabular}

Total traffic volume for the entire study period is $1,100,000$. 
52 St. SE between Peigan Trail SE and 130 Av. SE

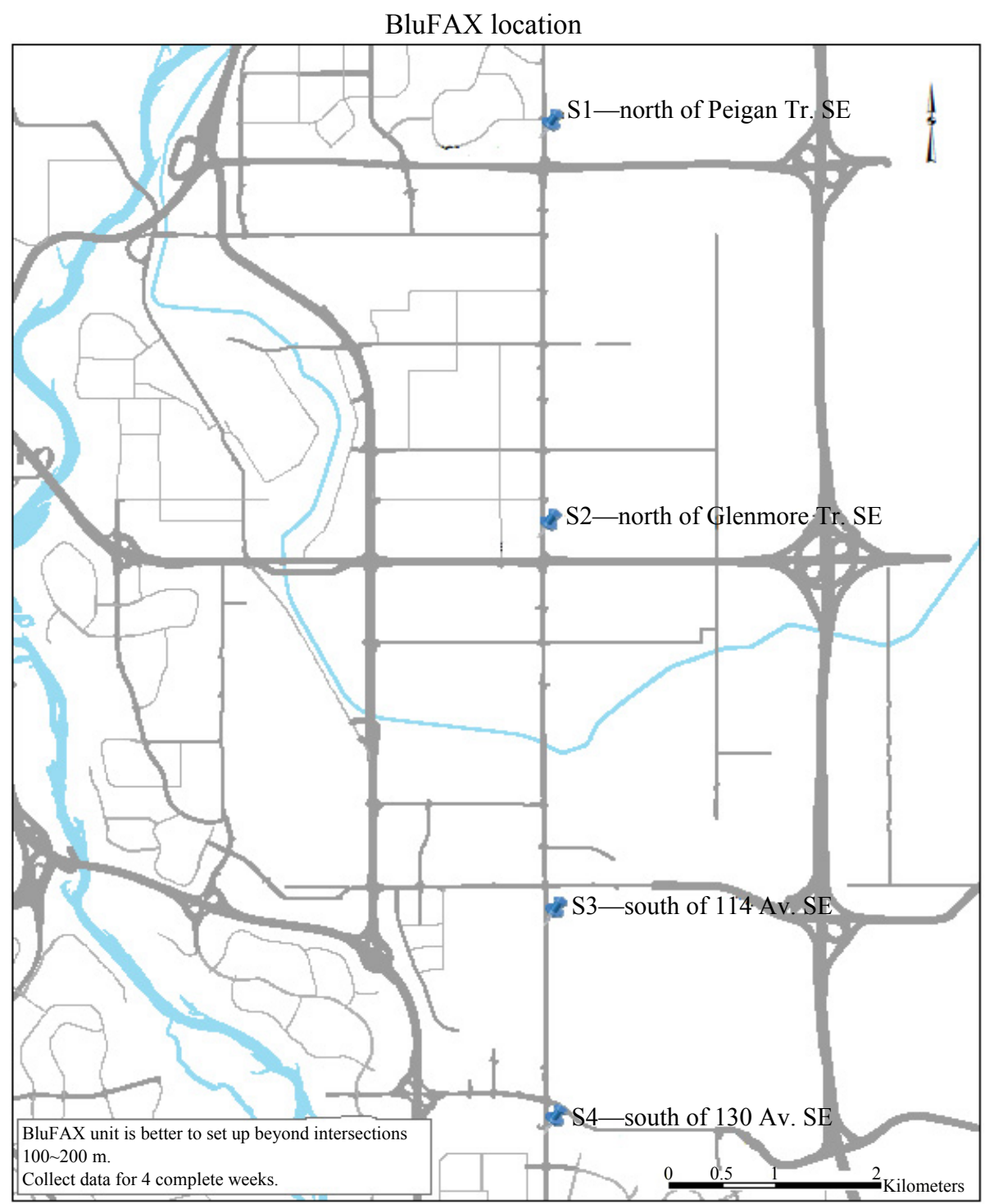

Fig. 8 The portable BluFAX sensors placement on 52nd St. in Calgary, Alberta.

Table 2 Comparative travel time reliability study on 52nd St. (24 h).

\begin{tabular}{llll}
\hline Indicator & BluFAX & TomTom & Difference \\
\hline Number of observations & 3434 & 960 & - \\
Detection rate & $2.3 \%$ & $0.6 \%$ & - \\
Weekday average travel time (min:s) & $11: 18$ & $11: 54$ & $5.25 \%$ \\
Weekday 95\% travel time (min:s) & $18: 18$ & $18: 36$ & $1.38 \%$ \\
Weekday buffer time (95th percentile-average travel time) (min) & $7: 00$ & $6: 36$ & $-5.56 \%$ \\
Weekday buffer index (\%) & $62.1 \%$ & $55.7 \%$ & $-11.41 \%$ \\
Weekday travel time index (\%) & $163.3 \%$ & $172.3 \%$ & $5.23 \%$ \\
Weekday planning time index (\%) & $264.7 \%$ & $268.3 \%$ & $1.34 \%$ \\
Weekday average speed (km/h) & 42.0 & 37.7 & $-11.31 \%$ \\
\hline
\end{tabular}

Total traffic volume for the entire study period is 150,000 . 
Table 1 summarizes the cumulative travel time reliability figures as well as speed between the 1st and the last station (S1-S4 southbound) on 52nd St. SE on working days over $24 \mathrm{~h}$.

Based on the BluFAX study outcome, the travel time reliability indices indicate that the drivers need to consider a significant extra travel time $(164.7 \%)$ to ensure an on time arrival. Similarly, TomTom indicates to $168.3 \%$ extra travel time to ascertain on time arrival. Regarding travel time index, the results show that the ratio of the actual travel time to the theoretical travel time for the case of BluFAX is estimated around 163.3\%. Like BluFAX, TomTom suggests $172.3 \%$ for the travel time index. Finally, both BluFAX and TomTom results show that the average speed on weekdays (42.0 kph and $37.7 \mathrm{kph}$, respectively) does not exceed $60 \%$ of the free flow speed which is $65 \mathrm{kph}$ on 52nd St.

Comparing the results of BluFAX and TomTom, the difference between travel time reliability figures, hovers between $5 \%$ and $10 \%$ : weekday planning time index (264.7\% vs. $268.3 \%)$ and weekday travel time index (163.3\% vs. $172.3 \%)$

Despite the inadequate number of TomTom observations on this corridor (only $0.6 \%$ ), the accuracy of results has significantly improved from the previous case study. This improvement evidently demonstrates that the number of observations plays an important role in accuracy of TomTom Traffic Stats. Nonetheless, further studies need to be done in order to: (1) verify the positive impact of the number of observations on the TomTom Traffic Stats accuracy; (2) determine the requirement of fulfilling a minimum penetration rate (comparable with that of suggested for using the Bluetooth technique) to leverage crowdsourcing techniques in travel time and speed studies.

\section{Conclusions and Final Remarks}

The main goal of this study was to examine the accuracy and applicability of traffic data from crowdsourcing techniques for travel time and reliability studies. The benchmark used in this study was Bluetooth traffic data source. We extracted and used the output of BluFAX sensors to record travel times over $24 \mathrm{~h}$ on working days on two major goods movement corridors (one freeway and the other one-a major arterial road) in Calgary in a 20-day time window. Similarly, in the context of crowdsourcing, we derived travel times from the TomTom self-service web portal, called Traffic Stats Portal, on the same corridors in the same study period. A series of customized TomTom historical traffic data were generated containing custom area analyses, travel times and speeds.

To calculate travel time reliability, we use travel time reliability metrics recommended by FHWA. The methodology is preferable since the calculated metrics are readily understandable by laypeople, including politician and the general public.

Our study results showed that the Bluetooth technology as the benchmark was able to provide reliable traffic data for the selected study corridors for short study periods. However, Bluetooth constraints and difficulty to analyze and process its generated massive data records in long study periods and particularly on heavily used roads can deter traffic analysts to count on this technology for such studies.

Crowdsourced data can be an appropriate alternative here. TomTom Traffic Stats Portal provides traffic data for the study area, although our case studies' results showed that the number of observations was much lower than that of BluFAX. In spite of this inadequacy, travel time indices on the arterial road were comparable to the baseline Bluetooth indices. Nevertheless, this finding cannot be interpreted as a general conclusion. Further researches need to be conducted to verify the accuracy of crowdsourcing technologies such as TomTom for travel time studies. Furthermore, it needs to be addressed whether a lower detection rate, compared to the $3 \%$ minimum penetration rate recommended by several sources for using the Bluetooth technology, can provide reliable estimates 
with a reasonable accuracy for travel time studies on Canadian roads.

\section{Acknowledgments}

The author wishes to acknowledge the help of Mr. Ekke Kok, the manager of Data Division in the City of Calgary for sharing his knowledge and making valuable comments, and traffic data analysts, David Zhong and Wendy Pan, for their important inputs and providing the required data for this study.

\section{References}

[1] The City of Calgary. 2013. Average Daily Weekday Traffic Volume Map. City of Calgary.

[2] Government of Alberta. 2010. "Alberta Transportation." Alberta Infrastructure and Transportation's Office of Traffic Safety. Accessed February 1, 2015. http://www.transportation.alberta.ca/.

[3] The City of Calgary. 2009. "Municipal Development Plan." City of Calgary. Accessed February 1, 2015. http://www.calgary.ca/mdp.

[4] The City of Calgary. 2009. "Calgary Transportation Plan." City of Calgary. Accessed February 1, 2015. http://www.calgary.ca/ctp.

[5] The City of Calgary, Transportation, Roads. 2014. "Truck Route Map.” Accessed January 1, 2014. http:/www.calgary.ca/Transportation/Roads/Pages/Truck -and-dangerous-goods/Truck-route-map.aspx.

[6] The City of Calgary. 2010. "Transportation Department." The City of Calgary. Accessed June 1, 2014. www.calgary.ca/TRANSPORTATION.

[7] Vo, T. 2011. "An Investigation of Bluetooth Technology for Measuring Travel Times on Arterial Roads: A Case Study on Spring Street.” M.Sc. thesis, Georgia Institute of Technology.

[8] Texas A \& M Transportation Institute. 2013. "Improved Data Collection for Improved Mobility." Transportation 2.0: Upgrading Our Transportation System 49 (4): 6-7.

[9] INRIX Traffic. "Home Page." 2014. INRIX Traffic. Accessed January 1, 2014. http://www.inrixtraffic.com/.

[10] Traffax Inc. 2014. "Manufacturer of BluFAX." Traffax Inc. Accessed March 1, 2014. http://www.traffaxinc.com/.

[11] Haghani, A., Hamedi, M., Sadabadi, K. F., Young, S. E., and Tarnoff, P. J. 2010. "Freeway Travel Time Ground Truth Data Collection Using Bluetooth Sensors." Transportation Research Record 2160: 60-8.

[12] MTO. 2012. Alternative Methodologies for Travel Time Studies. Final report.
[13] Szuch, C., McDaniel, Z., and Kok, E. 2011. "Measuring Goods Movement Travel Time and Reliability. Proposed Methodology." Presented at the 2011 Annual Conference of the Transportation Association of Canada, Edmonton.

[14] Prato, C. G., Rasmussen, T. K., and Nielsen, O. A. 2014. "Estimating Value of Congestion and Value of Reliability from the Observation of Car Drivers' Route Choice Behavior." Transportation Research Record 2412 (2412): 20-7.

[15] TomTom NV. 2014. "TomTom Traffic Index 2013." TomTom NV. Accessed November 1, 2013. http://www.tomtom.com/en_gb/trafficindex/.

[16] White, J. B. 2008. "New Services Gather Data in an Effort to Track Current and Future Traffic Jams." The Wall Street Journal. Accessed January 1, 2015. http://www.wsj.com/articles/SB12079509232400884.

[17] INRIX. 2015. “Analytics and SaaS Apps." INRIX. Accessed $\quad$ February 2015. http://www.inrix.com/Products.

[18] Ontario Ministry of Transportation. 2010. "Home Page." Ontario Ministry of Transportation. Accessed June 1, 2014. http://www.mto.gov.on.ca/english/.

[19] KMJ Consulting, Inc. 2010. Bluetooth Travel Time Technology Evaluation-Using the BlueTOAD. Pennsylvania: Haverford.

[20] KMJ Consulting, Inc. 2011. Using Bluetooth ${ }^{T M}$ to Measure Travel Time along Arterial Corridors: A Comparative Analysis. Pennsylvania: Haverford.

[21] The City of Calgary. 2015. "Roads Business Unit." The City of Calgary. Accessed February 1, 2015. www.calgary.ca/transportation/roads/pages/roads.asp.

[22] TomTom. 2014. "White Paper (2014), Historical Traffic Information." TomTom. Accessed March 1, 2014. http://www.tomtom.com/en_gb/licensing/products/traffic/ historical-traffic/custom-travel-times/.

[23] ITERIS. 2011. ITS Innovative Project: Arterial Travel Time Monitoring System Using Bluetooth Technology. Minnesota: Minnesota Department of Transportation.

[24] Young, S. 2008. "Bluetooth Traffic Monitoring Technology, Concept of Operation and Deployment Guidelines." University of Maryland Center for Advanced Transportation Technology. Accessed February 1, 2015. http://www.catt.umd.edu/sites/default/files/documents/U MD-BT-Brochure_REV3.pdf.

[25] Wassen, J. S., Sturdevant, J. R., and Bullock, D. M. 2008. "Real-Time Travel Time Estimates Using Media Access Control Address Matching." ITE (Institute of Transportation Engineers) Journal 78 (6): 20-3.

[26] Quayle, S., and Koonce, P. 2010. "Arterial Performance Measures Using MAC Readers-Portland's Experience.” Presented at North American Travel Monitoring 
Study to Calculate Travel Time Reliability in Calgary, Canada

Exposition and Conference 2010, Seattle, Washington.

[27] Office of Operations of the US Department of Transportation's Federal Highway Administration. "Travel Time Reliability: Making It There on Time, All the Time." Accessed May 1, 2013. http://www.ops.fhwa.dot.gov/publications/tt_reliability/tt r_report.htm.

[28] Ryus, P., Bonneson, J., Dowling, R., Zegeer, J., Vandehey, M., Kittelson, W., et al.. 2013. Proposed Chapters for Incorporating Travel Time Reliability into the Highway Capacity Manual. Washington D.C.: Transportation Research Board. 